\title{
Application of Liquid Phase Scanning Transmission Electron Microscopy Methods for the Quantitative Study of Membrane Proteins in Whole Cells
}

\author{
Diana B. Peckys ${ }^{1}$, Indra N. Dahmke ${ }^{2}$, Dalia Alansary ${ }^{1}$, Barbara Niemeyer ${ }^{1}$ and Niels de Jonge ${ }^{2,3}$ \\ 1. Department of Biophysics, Saarland University, Homburg/Saar, Germany. \\ 2. INM - Leibniz Institute for New Materials, Saarbrücken, Germany. \\ 3. Department of Physics, University of Saarland, Saarbrücken, Germany.
}

Membrane proteins are today's most important drugs targets. They reside at the interface between the environment and the interior of the cell, serving key messenger functions between the cell and the surrounding tissue and beyond. Methods are rare capable of studying this important class of proteins in their natural environment of the plasma membrane, while gaining data at the single-cell and singlemolecule level, due to diverse technical limitations. Within the past decade, liquid phase scanning transmission electron microscopy (Liquid STEM) has opened new perspectives to examine membrane proteins and nanoparticles (NP) in whole cells. Quantitative data can now be acquired from many tens of single cells and up to hundred thousand of single molecules within a matter of days. Compared to conventional electron microscopy methods for cells, involving dehydration, embedding and sectioning, the sample preparation for liquid phase STEM is comparatively fast and non-disruptive, similar as protocols used for fluorescence microscopy, thus minimizing the risk of artifacts. An important advantage is the option to perform correlative light and electron microscopy. Currently, three different methodologies are available to study intact, wet cells: 1) enclosing the sample in a microfluidic chamber inside a dedicated liquid flow holder, 2) imaging the cells in a saturated water vapor atmosphere in an environmental scanning electron microscope (ESEM) with an integrated STEM detector, and 3) coverage of wet cells with a graphene sheet [1], providing samples that fit all STEM systems.

The Liquid STEM method is based on the detection of small NP labels, specifically bound to the proteins of interest. Even in wet and micrometers-thick cellular samples, Liquid STEM can detect electron dense NP labels with a spatial resolution of 1-3 nm. Accurate analysis of the position, interaction, and oligomerization of the target protein requires a minimized distance between protein and bound NP, as well as a 1:1 label-to-target stoichiometry. The automatically detected label position coordinates of labeled target proteins can then be analyzed using different statistical methods, such as the pair correlation function, to distinguish true protein interactions from proteins at random proximity. This methodological principle can be used to examine, for instance, drug effects on the protein function at the molecular scale. For correlative microscopy, one uses labels made of electron dense- and fluorescent quantum dots. In addition, the parallel use of complementary fluorescent biomarkers allows the identification of specific cellular phenotypes in a heterogeneous cell population, as for instance in typical cancer cells. The latter approach permits to collect STEM data from identified, phenotype representative cells of rare subpopulations.

To illustrate how the three-different Liquid STEM methods can be applied to solve biological questions, we will present recent results involving two different membrane proteins. The first example, with implications for the urgent issue of drug resistance in cancer therapy, is about the distribution and conformation of the growth factor receptor HER2 in overexpressing cancer cell populations. This research is focusing on the molecular rearrangement HER2 experiences under the influence of HER2targeted antibody drug trastuzumab [2]. The second example, with major immunological significance, is 
about the determination of the ORAI1 ion channel stoichiometry [3]. This $\mathrm{Ca}^{2+}$ channel plays an essential role in intracellular $\mathrm{Ca}^{2+}$ homeostasis, indispensible for instance for $\mathrm{T}$-cell activation. Its stoichiometry under rest and in active state is a still debated topic. In addition, the possibility of cellular nanotoxicolgy studies [4] and the issue of imaging live cells [5] will also be discussed [6].

\section{References:}

[1] I N Dahmke et al, ACS Nano 11 (2017), p. 11108.

[2] DB Peckys et al, Mol Biol Cell 28 (2017), p. 3193.

[3] DB Peckys et al, Micros Microanal 22 (2016), p. 902.

[4] D Vanhecke et al, Beilstein J Nanotech 8 (2017), p. 2396.

[5] N de Jonge and DB Peckys, ACS Nano 10 (2016), p. 9061.

[6] The authors thank E. Arzt for his support through INM and the DFG through SFB1027.
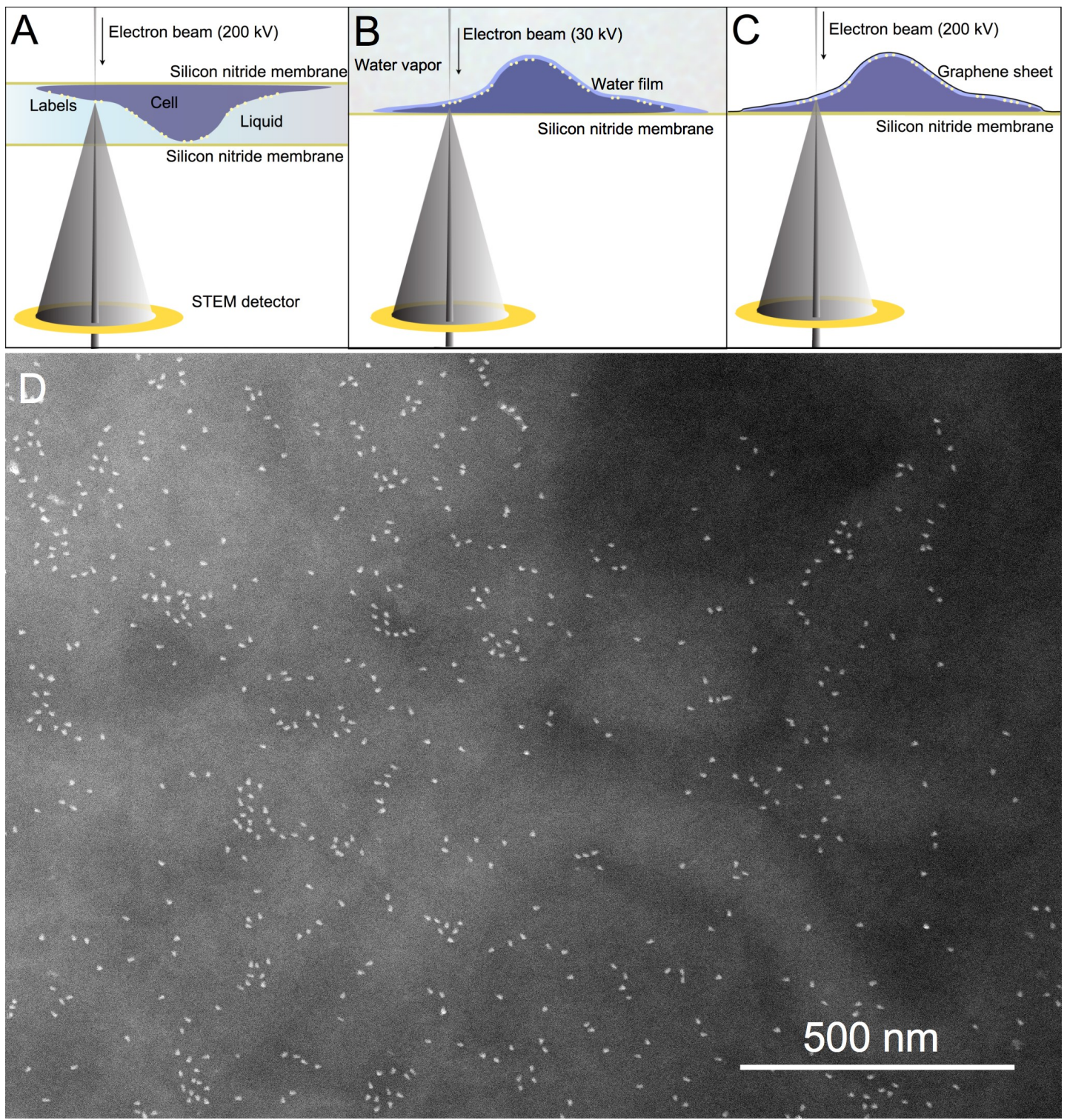

Figure 1. Three different options of Liquid STEM to study whole cell samples. (A) The sample can be imaged in a dedicated liquid flow holder, (B) in an ESEM with STEM detector or (C) covered with a graphene sheet, suitable for any STEM system. (D) Detail from a STEM image recorded with option (C) from a cell expressing HA-tagged ORAI1 proteins, after $\mathrm{Ca}^{2+}$ influx was initiated. ORAI1 oligomer formation is revealed by QD labels bound to the individual ORAI1 channel subunits. 